\title{
Study of English Translation and Its Application Based on Discourse Analysis
}

\author{
Mengchu Li ${ }^{1}$ \\ ${ }^{1}$ Chengdu Sport Institute, Chengdu, Sichuan, 610041
}

Keywords: discourse analysis; English translation; application

\begin{abstract}
Discourse analysis is a very important part of English translation. When reading, discourse analysis is very important. The analysis cannot only be based on words, sentences and words. It needs to be grasped in the original text. The paragraphs and themes are expressed. This is a means of expressing the author's thoughts. The analysis of discourse is very extensive. As the object of discourse analysis, it is necessary for the sentences, the collocation of the words, and the relationship of the whole article to be connected, which requires an in-depth understanding of the intent and purpose, an overall listing of language features, and a discourse analysis that emphasizes integration and plays an important role in improving their learning ability. Discourse analysis has important characteristics. When translating, it is necessary to understand the discourse center, master the entire central idea and paragraph structure of discourse, and analyze the context in a specific environment. Different discourses have different contexts, and their time, place, and characters need to be completed through discourse analysis. How to use the discourse analysis to translate the theory is the problem that is faced at present, and this paper mainly adopts the discourse analysis, selects the English translation, and uses the language structure to form the discourse.
\end{abstract}

\section{Introduction}

In English translation, the beginning of translation is a discourse analysis of the original English text. The traditional translation is mainly based on grammar. It does not further deal with the whole article, but simply emphasizes the understanding of words and sentences. The overall content, from the reading speed to the reading method, only emphasizes the proficiency of the sentence translation, does not understand the central theme of the article through translation, and has no practicality for translation, and the text translation mainly translates through integrity. Focusing on the whole article and translating from the convergence of the sentence, the article can be harmoniously unified from top to bottom, combined with context, to make translated articles more in line with requirements.

\section{The Essence of Discourse Analysis}

Discourse analysis is mainly used in education and teaching. At present, more and more attention is paid to the outside world. It is a translation that combines articles up and down, and is widely used in teaching. It starts with the central idea of the article and analyzes sentences. It is necessary to collaborate with and link paragraphs and phrases, to connect them through the main purpose of the article, to grasp the central idea, to understand the key explanations in grammar, to proceed from the context, and to combine the goals in teaching, and to strengthen it. Students take a holistic understanding of the article, break through the details, and develop the habit of reading through the text. Through semantic analysis, the use of clearer words and translation methods for translation, in the process of text analysis, is targeted. Students will be able to overcome the drawbacks of a leaf barrier, understand the characteristics of the paragraph in general and the structure of the entire article, and understand the logic between sentences and sentences.

Because the teaching community pays great attention to this method of discourse analysis, when it comes to a wide range of applications, especially in the process of English translation, it is necessary to permeate all the grammar and the subject of the article, so that the translation forms a 
certain degree of logic. Starting from the details of the article, it is important to identify the key points and important sentences. In order to better express the language and understand the whole sentence, this method can greatly increase the degree of understanding of students' translations. The point of view of translation and analysis is very beneficial to students.

\section{The Importance of Discourse Analysis in English Translation}

Discourse analysis is mainly based on the combination of external factors and intrinsic factors in English translation. After understanding the essence of discourse, it is necessary to understand the important role of this discourse in culture and the words that will meet the translation requirements. For translation, this is relatively simple. In the process of discourse analysis, it is necessary to analyze the characteristics of the source text and form a classification of comparative source texts. Through this classification, the source discourse analysis is used to make scenarios. In other words, translation is performed through the description of the situation. In the translation process, discourse changes may occur. This requires the combination of internal and external factors.

Discourse analysis enables translators to be more comprehensive in their translations. Translators can translate texts to meet translation requirements. In the process of translation, translations are translated according to the functions and framework of the translation. In this process, many factors determine translation. There are certain requirements on the function, and the features of the source text and the discourse structure play a certain role in translation. Under certain circumstances, the features of the source discourse need to be excavated and arranged according to functional requirements. At this time, translators need to make fact judgments, highlight the key points in the discourse, make decisions in the discourse that need to be deleted or made, make translations that need to be added or need to be corrected, and some do not A logical way of thinking requires proper and appropriate conclusions through textual translation.

Discourse analysis usually requires careful reading of articles and then translation. Although it takes more time and effort, the standardization of translation is relatively high. Professional discourse analysis takes less time than literary text analysis. When experienced translators analyze texts, their time will be relatively saved because these experienced translators will investigate through several key elements. In the translation teaching, discourse analysis is more important and its role is also very important. In the process of discourse analysis, especially the students who have just contacted the translation, they can improve their self-translation ability through this method. Many translations can Translating into them to study, the only way to use time as a vector to increase the intensity of translation learning.

The method of discourse analysis is more practical. From the perspective of discourse analysis, it plays an important role in translation. Whether it is professional or non-professional discourse and literary discourse, the main method is the same. The translator cannot have very great objectivity and will always incorporate the subjective color into his own consciousness. Because the translator is a more individualized individual, when translating, he needs to combine his own understanding with the article. For translation, its main point of view is to elaborate on the viewpoint of translation. It will further strengthen the translation ability through time learning. This requires discourse analysis as a front-line work, which is an indispensable translation method in translation.

\section{Discourse Analysis Theory and Information}

Discourse analysis is the extraction of the knowledge of multiple disciplines and will be used for specialized teaching and research communicative competence. Translation studies are the same as discourse analysis for translation. Its development and the 1960s, many theories are from Western countries. From the different perspectives, the method of discourse analysis has been summarized and entered into a profound analysis. The translated articles have been reorganized through the communicative sentences and have a certain foundation from the perspective of the translation strategy. This basis is Based on the original text, the emphasis on functional language is an important method of discourse analysis. Discourse analysis regards translation as a process of 
shallow depth. In this process, it is necessary to categorize the attributes and types of translated articles, use the communicative language usually used for translation, and perform discourse analysis through the body language structure. In the course of practice, it is necessary to reorganize the structure of the source text and learn from the top down.

The language learning of discourse is informative, and its powerful translation ability and analysis perspective play a very important role in translation studies. Discourse analysis is mainly through the inner-outside, top-down analysis of the difficult to solve the solution, the stylistic analysis of style, style, etc. in the discourse analysis of a unified theoretical solution, through sentences and sentences The convergence and the information in the article make the text an important feature. Analyze the context and intention, and translate better through the communication of discourse. The main theme and the mood of the article are to be figured out and the translated sentences are formed. Informationality is a centralized collection of textual analysis. This depends on the observation and subjective awareness of the communicators in the process of information transfer. When translators perform translation, the information transmitted has a certain significance. Fixed, but the value of the translation is different because of people. Information is also reflected in the use of language. The flexible use of language is of great significance to the recipient. The recipient interprets it. Whether it is the interpretation of the line of sight or the interpretation of the text, the process is subjective and subjective. The communicators exert their subjective consciousness and use a comprehensive language for translation. The translation content is analyzed layer by layer.

\section{Differences in English and Chinese Texts}

Discourse is mainly to connect a series of consecutive sentences into a whole language. According to research, it is confirmed that in the context of high context, the communication of information and the experience of the external environment are experienced, and the context is low, the display is clear, and the translation can be completed quickly. In English translation, the logical thinking is clear. The form of the article structure mainly consists of orderly arrangement of sentence clauses. It requires a more close connection. Its main idea is clear, grammar and function are relatively logical, and the arrangement and function of the sentences are more characteristic. However, English is a comparison. Low context level. The development of Chinese language from the logical thinking to the rigorous degree of the text is judged by the subjective consciousness. In the process of logical thinking, according to the requirements of the discourse, the coherent thoughts and the clear context are connected and the structure of the fish piece is constructed. To elaborate, with artistic conception and artistry, therefore, Chinese is a high-context discourse. When using Chinese to translate English, you need to pay attention to the connection and internal meaning of the article.

\section{Methods of English Translation from the Perspective of Discourse Analysis}

The method of discourse analysis can work well in English translation. The discourse is composed of sentences and words. It translates the meaning of its expression. It has more levels of expression and more complex ideas. It organizes several sentences. To become a phrase, to organize the expression segment and the next sentence, whether it is a single sentence or multiple sentences, all of which are of independent significance, have the function of linking up with one another, and at the same time have a close connection. Therefore, multiple Discourse is formed by the combination of segments,

The segments that make up the discourse are of different types, but are closely related to the discourse. From different perspectives, to analyze discourse topics, you can raise the understanding of words and sentences to a level, import them from the meaning of the words and explanations of words, and sort out the overall meaning and framework of the article. From top to bottom Translation, sentence translation and word translation are used for topic analysis. Levels are introduced from the level of the article. Deep understanding of the content of the article enables 
good translation.

The translation of the article is not simply a translation of the framework and the meaning, but rather the translation through a natural paragraph. The natural paragraph is more common in the article, with the title and the text, through different forms of natural paragraphs and layers, the natural paragraphs of different levels are arranged in order, and they form articles by linking. Articles composed of different levels are also different, and their logical thinking relationships are not the same. When the topics are revolved, they form. A few paragraphs of an article, the article is higher than the level, the level is higher than the natural segment, this semantic structure is conducive to thinking of the theme as a unity, the formation of a close connection between the paragraphs, the article from above to the next translation, there is more than one kind of vector to link the language form. Therefore, when translating the article, it is necessary to clarify the subject first, and through the analysis of layers of paragraphs, a better article will be translated.

As the main body of translation, a large number of textual materials need to be read through translation. The discourse is a kind of object of translation. It mainly has the ability to bear, and its essence has been separated from the cultural context. It needs to have a source context. To interpret correctly, it is necessary to practice the analysis of the cultural context it produces. The translator needs to familiarize with the cultures of various places before they can perform a good translation. Discourse is an integral part of social culture and context. In the process of translation, information is accepted and the language of translation culture is refined. The discourse also covers a large number of disciplines and has certain cultural and historical factors. Its cultural differences can be seen everywhere.

\section{Conclusion}

From the perspective of discourse analysis, English can be well translated. On the basis of focusing on the analysis of discourse, it is necessary to carry out layers of articles and articles from top to bottom, because of cultural differences, English translation There are certain differences in the logical thinking of becoming Chinese. The method of discourse analysis grasps the structure of the article as a whole, combines the language features and context, and translates the English translation in detail.

\section{References}

[1] Qiu Xingxin. An analysis of the application of discourse analysis theory in college English reading teaching [J]. English teacher, 2015, 1524:16-17.

[2] Luo Bingyan. Analysis of the role of discourse analysis in college English teaching [J]. Science Consulting (Technology • Management), 2016, 07:104.

[3] Tao Yaning, Li Shiping, Hu Ping. The application of discourse analysis in classical translation and translation criticism [J]. Overseas English, 2011, 08: 232-233.

[4] Wu Ni. Application of discourse analysis in translation studies [J]. Overseas English, 2011, 12: 202-203.

[5] Wang Xueling. Application of Discourse Analysis Theory in Translation Teaching [J]. Journal of Xinxiang University, 2014, 3103:28-30.

[6] Zhu Xiaoyi. Discourse Analysis and the Development of Non-English Major Postgraduates' Text Translation Competence [J]. Chinese School Education, 2009, 10:48-49.

[7] Chen Xi. From Functional Discourse Analysis to Translation Teaching [J]. Foreign Languages in China, 2012, 901:94-97+111.

[8] Li Qiaoqiao, Liao Can. Discourse Analysis and Translation Studies [J]. Journal of Inner Mongolia Agricultural University (Social Sciences Edition), 2012,1405:353-355. 
[9] Bai Yujing. Application of Translation Techniques from the Perspective of Discourse Analysis [J]. Journal of Mudanjiang Institute of Education, 2015, 04:21-22.

[10] Yan Xiaoru. Application of discourse analysis in the teaching of intensive reading in English majors [J]. The Journal of Science and Education (first edition), 2015, 08: 81-83.

[11] Liu Qi. Discourse analysis plays an important role in college English intensive teaching [J]. Journal of Sichuan College of Education, 2008, S1: 75-76.

[12] Zheng Yichang. The Cohesion and Coherence in Chinese-English Translation from the Perspective of Discourse Analysis Theory [J]. Journal of Hebei North University, 2005, 05:22-24. 